\section{Balloon occlusion of pharyngeal pouch as aid to nasoenteric intubation}

Nasoenteric feeding plays an important part in the nutritional support of debilitated patients. ${ }^{\prime}$ Nevertheless, attempts to introduce the catheter in a patient with a pharyngeal pouch may be difficult; we describe a way of overcoming this, using a balloon to occlude the neck of the pouch.

\section{Case report}

An 82 year old man who had had a previous stroke affecting his right side was admitted with left sided weakness, difficulty in swallowing, and dysarthria. The difficulty in swallowing had developed two days before admission, with resultant

preferentially directed into the oesophagus (figure). With the enteric tube now distal to the pharyngeal pouch the pouch was aspirated through the Foley catheter, the balloon was deflated and removed, and positioning of the enteric tube in the proximal small bowel continued as normal.

The patient was nourished nasoenterically for 12 days. With partial neurological recovery the tube was removed. The pouch was treated with an endoscopic Dohlman procedure, and six months after the second stroke the patient remained independent.

\title{
Comment
}

As in this case the neurological deficit from a stroke may improve with time, and efforts should be made to support such patients nutritionally if recovery is expected. Oesophagopharyngeal pouches are not uncommon, and if pharyngeal dyscoordination is also present the only method previously
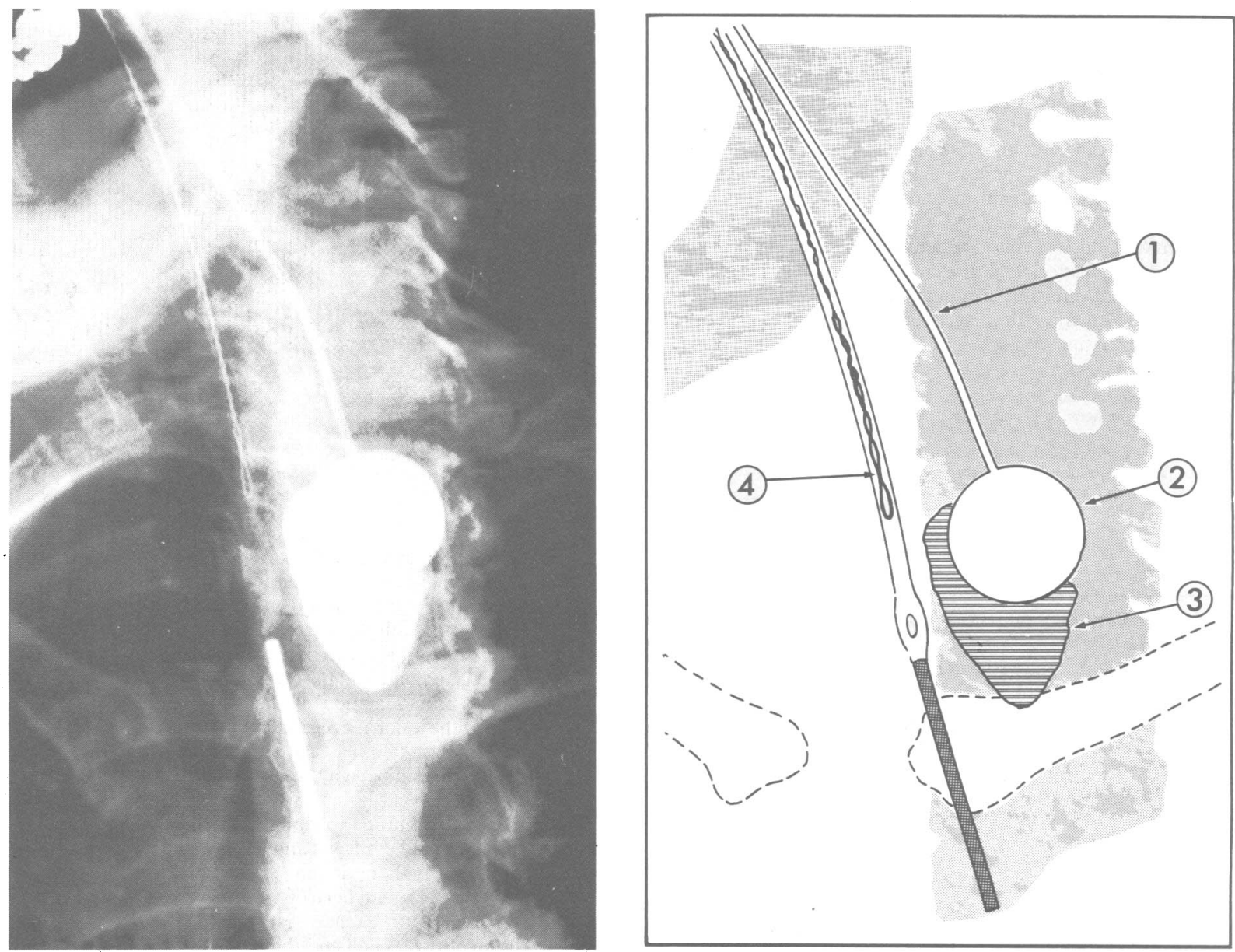

Radiograph and diagram of occluded pharyngeal pouch with nasoenteric tube passed beyond it. (1) Foley catheter; (2) Foley catheter balloon; (3) contrast medium in pharyngeal pouch; (4) nasoenteric tube with guide wire passing into oesophagus.

regurgitation of all liquids and solids and some aspiration into the trachea and bronchi. Several unsuccessful attempts were made by nursing and medical staff to pass a nasogastric tube. Under general anaesthesia further unsuccessful attempts to introduce the tube were made with an endoscope. Eventually, under the same anaesthetic, the tube was passed by using a laryngoscope. Shortly afterwards he regurgitated the tube and subsequently developed an aspiration pneumonia. Once again endoscopic introduction of the tube was unsuccessful, and it was suggested that future feeding would have to be through a gastrostomy. The oesophagus was examined with barium; there was pharyngeal dyscoordination with a large pharyngeal pouch, into which swallowed material passed preferentially, thence overflowing into the airway.

To occlude the pouch a 12 French gauge Foley catheter was inserted under fluoroscopic control into the hypopharynx through the nostril. It passed preferentially into the pharyngeal pouch, whose position and size were assessed by instilling some dilute barium through the catheter. The catheter balloon was then inflated with a dilute water soluble contrast medium ( $3 \mathrm{ml}$ of $25 \%$ Hypaque) and gently drawn back until the pouch neck was occluded.

An 8 French gauge $109 \mathrm{~cm}$ Corsafe enteric feeding tube with a tungsten weighted tip ( $E$ Merck Ltd) was passed through the other nostril; when it reached the region with the pharyngeal pouch gentle traction was put on the Foley catheter while the nasoenteric tube was advanced. The rounded end of the nasoenteric tube then glanced off the inflated Foley catheter balloon and was available of guiding a tube into the oesophagus avoiding the pouch was endoscopic. The endoscopic method is difficult, uncomfortable for the patient, and risks perforation of the wall of the pouch, which is composed only of mucosal and submucosal layers. Our new technique is cheap, safe, and easily performed in a radiological department.

We thank Dr Richard Harvey for his suggestions and encouragement, and the department of medical illustration, Frenchay Hospital, for the illustrations.

1 Silk DBA. Enteral nutrition. Postgrad Med f 1984;60:779-90.

(Accepted 5 March 1987)

Department of Radiology, Frenchay Hospital, Bristol BS16 1LE

I W J BIRCHALL, MRCP, FRCR, registrar

R L LAW, DCR, superintendent radiographer

Correspondence to: Dr I W J Birchall, Department of Radiodiagnosis, Bristol Royal Infirmary, Bristol BS2 8HW. 